\title{
Electron confinement in single layer graphene quantum dots: semiclassical approach
}

\author{
G. Giavaras ${ }^{\mathrm{b}}$, P. A. Maksymª, M. Roy ${ }^{\mathrm{a}}$ \\ ${ }^{a}$ Department of Physics and Astronomy, University of Leicester, Leicester LE1 7RH, UK \\ ${ }^{b}$ Department of Materials, University of Oxford, Oxford $\mathrm{OX} 13 \mathrm{PH}, \mathrm{UK}$
}

\begin{abstract}
A semiclassical approach is used to investigate the physics of electrons confined in a graphene quantum dot in a magnetic field. The states near the gap in the spectrum are localised between the turning points for classical massless particles. This fact is used to obain approximate energies and states in the case of non-zero, positive angular momentum. The agreement with exact numerical energies is about $1.5 \%$ or better and the nodal structure of the exact states is predicted correctly. The exact and approximate quantum states also agree well. The approach is most accurate in the regime of large angular momentum, large magnetic field and small radial quantum number.
\end{abstract}

Key words: Graphene, Quantum Dot

PACS: 73.61.Wp, 73.21.La

\section{Introduction}

Graphene charge carriers are massless and this affects their dynamics quite dramatically. In contrast to semiconductors whose charge carriers have mass, an external potential applied to a single layer of graphene cannot confine electrons. So it is not possible to fabricate a quantum dot by applying an external potential to graphene: any electrons in the dot eventually tunnel out and this is a manifestation of the quantum Klein paradox $[1,2]$. This results in quasi-confined states $[3,4]$ that are similar to scattering resonances. However it has recently been shown that it is possible to confine electrons with a combination of electric and magnetic fields [5]. The magnetic field gives the dot states an exponentially decaying tail while the electrostatic potential defines the centre around which the state is localised. Confinement in this situation is conditional and the conditions for confinement are derived exactly in ref. [5]. These conditions depend on both the form of the potential and its parameters. Interestingly, there are potentials for which the confinement is experimentally tunable, that is the tail of the dot wave function can be changed from exponentially decaying to oscillatory just by varying the strength of the potential or the magnetic field. Further, the same effect occurs in a realistic model of a graphene $\operatorname{dot}[5]$.

The quantum states studied in ref. [5] were computed numerically but it is desirable to have analytic solutions to obtain maximum insight into the physics. Although there are very few cases where the two-component radial equations for a graphene dot can be solved exactly, it is possible to make some approximations that describe the confined states accurately. The idea is to find the region where the state has large amplitude and approximate the radial equation in this region so that it can be solved analytically. This demonstrates that the states tend to be localised in the region between classical turning points for massless particles and explains the nodal structure of the ex- act states. The present work is focused on states of non-zero, positive angular momentum where there are particularly simple expressions for the energy levels.

\section{Semiclassical Approach}

The dot model is the same as the one in ref. [5]. The scalar potential is $V=V_{0} r^{s}$ where $r$ is the radial distance, the magnetic field is perpendicular to the graphene sheet and the azimuthal component of the vector potential is $A_{\theta}=A_{0} r^{t}$, where $s, t>0$. Because of circular symmetry the quantum states are eigenstates of angular momentum, $\hbar m$. The two-component envelope function is $\left(\chi_{1}(r) \exp (i(m-1) \theta), \chi_{2}(r) \exp (\operatorname{im} \theta)\right)$ where $\theta$ is the azimuthal angle. The radial functions, $\chi_{i}$ satisfy [5]

$$
\begin{aligned}
\frac{V}{\gamma} \chi_{1}-i \frac{d \chi_{2}}{d r}-i \frac{m}{r} \chi_{2}-i \frac{e}{\hbar} A_{\theta} \chi_{2} & =\frac{E}{\gamma} \chi_{1}, \\
-i \frac{d \chi_{1}}{d r}+i \frac{(m-1)}{r} \chi_{1}+i \frac{e}{\hbar} A_{\theta} \chi_{1}+\frac{V}{\gamma} \chi_{2} & =\frac{E}{\gamma} \chi_{2},
\end{aligned}
$$

where $\gamma=646 \mathrm{meV} \mathrm{nm}$ and $E$ is the energy.

The first step in the semiclassical approach is to symmetrize the angular momentum terms and make the equations real by putting $f_{1}=\sqrt{r} \chi_{1}, i f_{2}=\sqrt{r} \chi_{2}$. This gives

$$
\begin{aligned}
\frac{V}{\gamma} f_{1}+\left(\frac{d}{d r}+\frac{\left(m-\frac{1}{2}\right)}{r}+\frac{e}{\hbar} A_{\theta}\right) f_{2} & =\frac{E}{\gamma} f_{1}, \\
\left(-\frac{d}{d r}+\frac{\left(m-\frac{1}{2}\right)}{r}+\frac{e}{\hbar} A_{\theta}\right) f_{1}+\frac{V}{\gamma} f_{2} & =\frac{E}{\gamma} f_{2},
\end{aligned}
$$

where the physical significance of $f_{i}$ is that $f_{i}^{2}$ gives the radial probability distribution for each component.

The next step is to decouple the equations approximately. Putting $P=f_{1}+f_{2}, M=f_{1}-f_{2}$ and differentiating each equa- 
tion with respect to $r$ eventually leads to

$$
\begin{aligned}
& c^{2} \hbar^{2} \frac{d^{2} P}{d r^{2}}+c^{2} p_{r}^{2} P-(E-V+U)^{\prime} M=0, \\
& c^{2} \hbar^{2} \frac{d^{2} M}{d r^{2}}+c^{2} p_{r}^{2} M+(E-V-U)^{\prime} P=0,
\end{aligned}
$$

where prime denotes differentiation with respect to $r, c=\gamma / \hbar$, $U=c\left(\hbar\left(m-\frac{1}{2}\right) / r+e A_{\theta}\right), p_{r}^{2}$ is the classical radial momentum and $c^{2} p_{r}^{2}=(E-V)^{2}-U^{2}$. Eqs. (1) and (2) decouple approximately when $(E-V \pm U)^{\prime}$ is small and the conditions for this to be valid are found by considering the classical motion of massless particles.

The classical motion is restricted to the region where $c^{2} p_{r}^{2}>$ 0 and, provided that the state is confined, $c^{2} p_{r}^{2}$ is stationary at only one point and this stationary point is a maximum [5]. While this is true for all values of $m$, the properties of the classical turning points depend on $m$. The turning points are the two roots of $c^{2} p_{r}^{2}=(E-V+U)(E-V-U)=0$ and when $m>0$ both roots come from one of the factors. It is this property that allows approximate energies to be found simply. When $m \leq 0$ a different and more complicated approach is needed [6].

In the case when $m>0$, either $E-V-U$ has a maximum between the two classical turning points or $E-V+U$ has a minimum. Fig. 1 illustrates the case when $E-V-U$ has a maximum. The magnetic field is uniform, $B=1.1 \mathrm{~T}, V=V_{0} r$ with $V_{0}=0.5 \mathrm{meV} \mathrm{nm}^{-1}, E=165 \mathrm{meV}$ and $m=10$. For clarity, $c^{2} p_{r}^{2}$ is scaled by a factor of $1 / 7$. The maximum of $E-V-U$ occurs between the two classical turning points and is close to the maximum in $c^{2} p_{r}^{2}$ but does not coincide with it. These properties of $c^{2} p_{r}^{2}$ and its factors allow Eqs. (1) and (2) to be simplified. For example, when $E-V-U$ has a maximum, $(E-V-U)^{\prime} \sim 0$, Eq. (2) is satisfied by $M \sim 0$ and Eq. (1) reduces to

$$
c^{2} \hbar^{2} \frac{d^{2} P}{d r^{2}}+c^{2} p_{r}^{2} P=0 .
$$

In this situation $f_{1} \sim f_{2}$ and exact numerical results (Section 3)show that this approximation is very good, particularly at large $m$. Similarly there are states for which $f_{1} \sim-f_{2}$ and $M$ is found approximately by replacing $P$ in Eq. (3) by $M$.

When the distance between the classical turning points is small, the quantum state is localised close to the maximum in $c^{2} p_{r}^{2}$ and the solution to Eq. (3) can be obtained approximately by replacing $c^{2} p_{r}^{2}$ by its Taylor expansion about the maximum at $r=r_{\text {max }}$, that is $c^{2} p_{r}^{2} \sim F_{0}-\frac{1}{2} \beta\left(r-r_{\text {max }}\right)^{2}$ where $F_{0}$ is the value of $c^{2} p_{r}^{2}$ at $r_{\max }$ and $\beta$ is the value of the second derivative. Substituting this form into Eq. (3) leads to the harmonic oscillator equation of non-relativistic quantum mechanics and the requirement that the quantum states do not diverge leads to solutions that have the same functional form as those for the non-relativistic oscillator. The non-divergent solutions are found when $F_{0}$ takes quantised values given by

$$
F_{0}(E)=\hbar c \sqrt{2 \beta(E)}\left(N+\frac{1}{2}\right),
$$

where $N \geq 0$ is an integer quantum number equal to the number of nodes in the state. Eq. (4) gives $E$ implicitly and can be

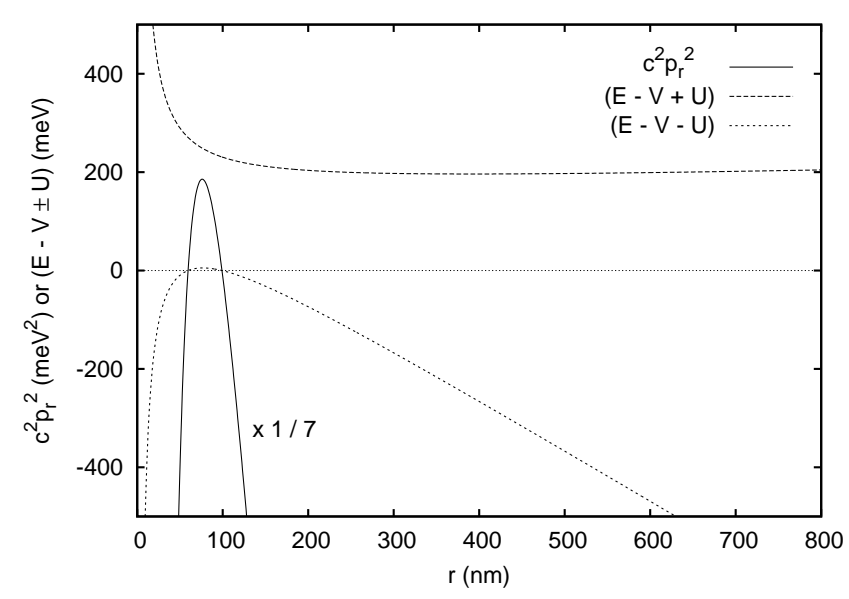

Figure 1: Typical behaviour of $c^{2} p_{r}^{2}$ and its factors.

solved numerically to find the approximate energy of the state with $N$ nodes.

It is also possible to find an explicit expression for the energy with the aid of another approximation. This makes use of fact that $r_{\max }$ nearly coincides with the maximum in $(E-V-U)$ as illustrated in Fig. 1. The position of the maximum in $c^{2} p_{r}^{2}$ is given by $\left(c^{2} p_{r}^{2}\right)^{\prime}=(E-V-U)^{\prime}(E-V+U)+(E-V-U)(E-V+$ $U)^{\prime}=0$. When the region between the classical turning points is narrow, $(E-V-U)$ remains small between the turning points and when $(E-V+U)^{\prime}$ is not too large the condition for the maximum is approximately $(E-V-U)^{\prime}=0$. Therefore $r_{\text {max }}$ is close to $\tilde{r}_{\text {max }}$, the position that maximises $(E-V-U)$.

This allows Eq. (4) to be solved because $\tilde{r}_{\text {max }}$ is independent of $E$, while $r_{\text {max }}$ depends on $E$. Replacing $r_{\max }$ with $\tilde{r}_{\max }$ in Eq. (4) results in

$$
(E-\tilde{V})^{2}=\tilde{U}^{2}+\hbar c \sqrt{2 \tilde{\beta}}\left(N+\frac{1}{2}\right),
$$

where $\tilde{U}, \tilde{V}$ and $\tilde{\beta}$ are evaluated at $r=\tilde{r}_{\text {max }}$. The energy is given by

$$
E=\tilde{V}+\left[\tilde{U}^{2}+\hbar c \sqrt{2 \tilde{\beta}}\left(N+\frac{1}{2}\right)\right]^{\frac{1}{2}},
$$

where the positive root is taken because $(E-\tilde{V})>0$ between the turning points.

The states with $f_{1} \sim f_{2}$ therefore correspond to a ladder of levels whose energy increases with $N$. Similarly, it can be shown that the states with $f_{1} \sim-f_{2}$ correspond to a ladder of levels whose energy decreases with $N$. There is a gap between these ladders because there is a gap between the two factors of $c^{2} p_{r}^{2}$ as can be seen in Fig. 1. Consequently there is an energy range where neither factor has a root, classical motion is not allowed and corresponding quantum states do not occur. Fig. 1 shows the situation when the classical energy is just above the threshold for the increasing ladder. Decreasing this energy by a few meV shifts $(E-V \pm U)$ down slightly and puts the system in the gap. In some situations, for example when $V=V_{0} \sqrt{r}$, and the magnetic field is small, the gap is small and there is some 


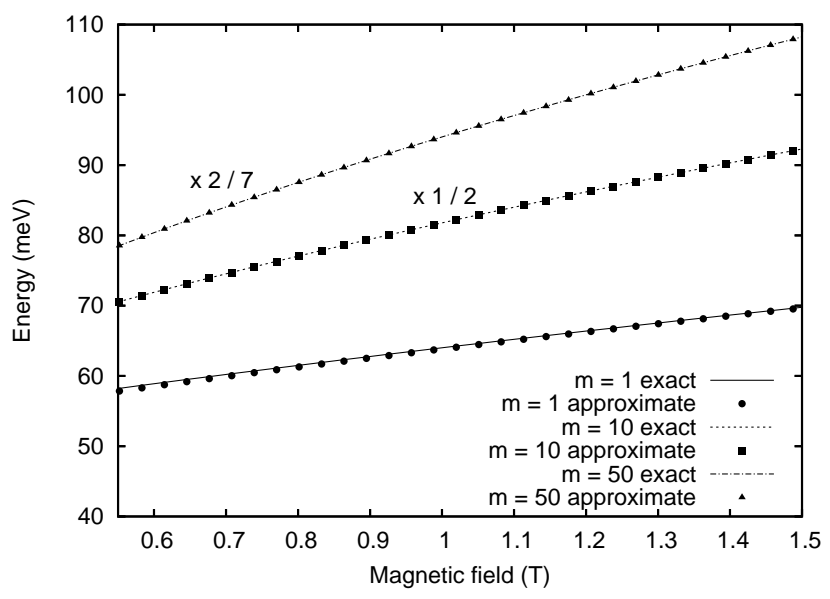

Figure 2: Exact and approximate energies for $V=V_{0} \sqrt{r}$.

tendency for the states associated with each ladder to overlap. These considerations explain the qualitative form of the numerically calculated spectrum. In addition, the number of nodes given by the quantum number $N$ agrees exactly with the number of nodes found in the numerically calculated states. The quantitative accuracy of the approximations is discussed in the next section.

The present approach is closely connected to the exact analysis in ref. [5]. In ref. [5] the coupled equations for the twocomponent radial function are decoupled exactly and reduced to an equation of the form $u^{\prime \prime}+k^{2}(r, E) u=0$. The character of the states, that is whether they are confined or de-confined, is then found by considering the asymptotic sign of $k^{2}$. This coefficient is a complicated function of $r$ and $E$ but its physical meaning is that $\hbar^{2} k^{2}$ is equal to $p_{r}^{2}$ plus quantum corrections. The neglect of these corrections is the essence of the present approach and the analysis in this section shows this should be valid when the angular momentum is positive and the classically allowed region is not too broad.

\section{Comparison with Exact Results}

The semiclassical approach is only valid for confined states but electron confinement in a graphene dot is conditional. The exact and approximate energies and wave functions are compared in this section for several cases where the states are known to be confined. The general analysis of ref. [5] shows that states are always confined in a magnetic field provided that $s<t$. For a uniform field this requires $s<1$ and the case of $s=0.5$ is the first test case considered here. Confinement is also possible when $s=t$. In this case confinement depends on the strength of the magnetic field and scalar potential and a confinement-deconfinement transition occurs at the critical field strength where $V_{0}^{2}=c^{2} e^{2} A_{0}^{2}$. The second test case considered here is when $s=t=1$ and the uniform magnetic field is on the confined side of the transition. The exact energies and states for these cases are computed with the method outlined in ref. [5].

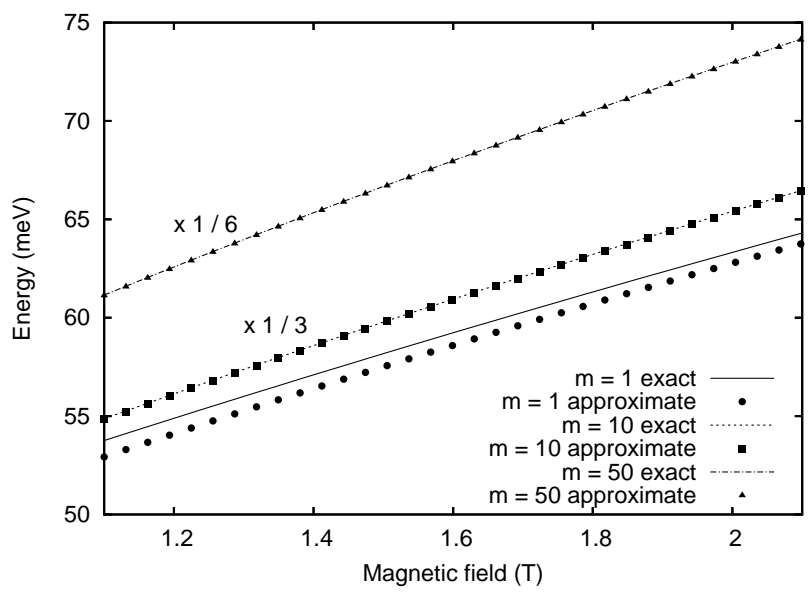

Figure 3: Exact and approximate energies for $V=V_{0} r$.

Fig. 2 shows exact and approximate energies for the nodeless state at the bottom of the increasing ladder of levels. The potential is $V=V_{0} \sqrt{r}$ with $V_{0}=5 \mathrm{meV} \mathrm{nm}^{-1 / 2}$. The approximate energies are calculated from Eq. (6). Similar energies may be obtained from Eq. (4) and for $m=10$ and $m=50$ these two energies agree to four figures. The agreement with the exact energies is also excellent, typically around $0.03 \%$ for $m=10$ and $m=50$. However for $m=1$ there are discrepancies. The energies given by Eqs. (4) and (6) differ by $3-11 \%$. The exact energy lies between these two values and is closest to the value given by Eq. (6). The worst discrepancy between this value and the exact energy is around $0.7 \%$ at $m=1, B \sim 0.55 \mathrm{~T}$. The agreement generally improves as $m$ or $B$ increase. The reason for this is that the distance between the classical turning points decreases with $m$ or $B$. The approximation that $f_{1} \sim f_{2}$ then improves. The approximation is also found to get worse as $N$ increases and the reason for this is the same. Any increase of $N$ takes the energy away from the gap in the spectrum and this is accompanied by an increase in the distance between the turning points.

The exact and approximate energies for $V=V_{0} r, V_{0}=0.5$ $\mathrm{meV} \mathrm{nm}{ }^{-1}$ are shown in Fig. 3. The approximate energies are again calculated from Eq. (6). The approximation is slightly less accurate than for the $\sqrt{r}$ potential but the results show the same trends. In particular, the accuracy of the approximate energies improves when $m$ or $B$ increase. The worst discrepancy is about $1.5 \%$ at $m=1$ and $B=1.1 \mathrm{~T}$ while the best is about $0.04 \%$ at $m=50$ and $B=2 \mathrm{~T}$. These trends can again be explained by considering the distance between the turning points.

Fig. 4 shows exact and approximate states corresponding to the energies shown in Fig. 3. For clarity the centre of the $m=10$ states is displaced by $100 \mathrm{~nm}$ to the right and the centre of the $m=50$ states is similarly displaced by $200 \mathrm{~nm}$. The square of each state is shown and the magnetic field is $1.6 \mathrm{~T}$. It is clear that the two components approach each other as $m$ increases. The approximate state is always localised close the the exact states but for $m=1$ it is slightly too narrow. For large $m$ the accuracy improves but the approximation does not reproduce the peak 


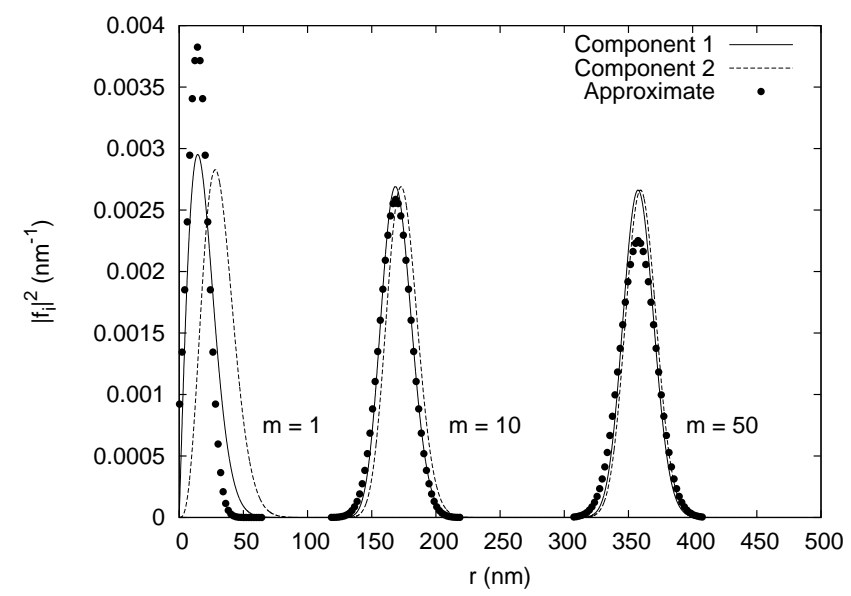

Figure 4: Exact and approximate states for $V=V_{0} r$.

heights exactly. The reason appears to be that the exact peak is not symmetric, while the approximate one is. The effect of this is to transfer weight from the centre of the state to its tail, resulting in a reduction of the peak height. This is particularly clear when $m=50$.

\section{Discussion}

A semiclassical approach has been used to illustrate the close link between the classical physics of massless particles and the properties of graphene dot states. Confined states for positive $m$ tend to be localised between the two classical turning points for massless particles and the two wave function components are approximately the same or differ in sign. This allows the energies and quantum states to be found approximately, explains why there are two ladders of energy levels separated by a gap and predicts the number of nodes in each state correctly. For large $m$, the approximate and exact aenergies agree to $1.5 \%$ or better.

\section{Acknowledgements}

This work was supported by the UK Engineering and Physical Sciences Research Council. PAM is grateful for study leave from the University of Leicester and hospitality from Prof. H. Aoki, Department of Physics, University of Tokyo where this work was written.

\section{References}

[1] M. I. Katsnelson, K. S. Novoselov and A. K. Geim, Nature Phys. 2, 620 (2006).

[2] A. K. Geim and K. S. Novoselov, Nature Materials 6, 183 (2007).

[3] Hong-Yi Chen, Vadim Apalkov and Tapash Chakraborty, Phys. Rev. Lett. 98, 186803 (2007).

[4] A. Matulis and F. M. Peeters, Phys. Rev B 77, 115423 (2008).

[5] G. Giavaras, P. A. Maksym and M. Roy, J. Phys. Condens. Mat. 21, 102209 (2009).

[6] G. Giavaras, P. A. Maksym and M. Roy, in preparation. 